\title{
THE POINCARÉ DUAL OF THE WEIL-PETERSSON KÄHLER TWO-FORM
}

\author{
ROBERT C. PENNER
}

\begin{abstract}
We consider a family of conjectures due to Witten which relate the Miller-Morita-Mumford cohomology classes to explicit cycles in a certain cell-decomposition of the moduli space of punctured Riemann surfaces. A version of the first of these conjectures is proved here, and the computational geometric proof leads directly to Rogers' version of the dilogarithm and its Abel-Spence functional equation.
\end{abstract}

\section{INTRODUCTION}

Let $\mathcal{M}=\mathcal{M}_{g}^{s}$ denote the uncompactified moduli space of Riemann surfaces of genus $g$ with $s \geq 1$ punctures (so the real dimension of $\mathcal{M}$ is $N=6 g-6+2 s$ ), and let $\overline{\mathcal{M}}$ denote the Deligne-Mumford compactification $[\mathrm{DM}]$ of $\mathcal{M}$, which is an orbifold again of dimension $N$. Recall from [Mi], [Mo], [Mu] the (rational) Miller-Morita-Mumford cohomology classes $\bar{\kappa}_{i} \in H^{2 i}(\overline{\mathcal{M}} ; Q)$, for $i \geq 1$, which are stable classes in the sense of [Ha]. Of course, the class $\bar{\kappa}_{i}$ pulls back under the inclusion $\mathcal{M} \subset \overline{\mathcal{M}}$ to a well-defined class $\kappa_{i} \in H^{2 i}(\mathcal{M} ; Q)$, for $i \geq 1$, and we refer to these classes as the Miller-Morita-Mumford classes on $\mathcal{M}$

This paper is dedicated to a family of conjectures by Edward Witten [private communication] about the Poincaré duals of the Miller-Morita-Mumford classes on $\mathcal{M}$. Before discussing the conjectures, we must briefly discuss some background material.

Recall (see [BE], [Ha], [P1], [P3]) that $\mathcal{M}$ comes equipped with a canonical "cell-decomposition"; namely, there is an explicit decomposition of $\mathcal{M}$ into regions, each of which is naturally homeomorphic to the interior of a simplex together with certain of its faces. The "cell-decomposition" does not, so far

Partially supported by the National Science Foundation 
as we know, extend reasonably to a true cell-decomposition of $\overline{\mathcal{M}}$. Thus, we shall be forced to consider cycles of non-compact support in our "celldecomposition" of $\mathcal{M}$ which are expressed as rational linear combinations of interiors of simplices in this "cell-decomposition".

In fact, Witten describes for each $N \geq 2 i \geq 2$ an explicit closed rational $(N-2 i)$-cycle $\left[W_{2 i}\right]$ of non-compact support in our "cell-decomposition" of $\mathcal{M}$ and makes the

Conjecture 0.1 (Witten). $\kappa_{i}$ is a constant multiple of the Poincare dual of $\left[W_{2 i}\right]$ on $\mathcal{M}$, for each $i \geq 1$.

Remark 0.1. Witten proposed a proof of these conjectures using Chern-Weil theory, and there is actually a natural section to the fiberwise cotangent bundle of the universal curve in the current context. A difficulty with this approach, however, is that the natural section is not generic, and this renders the calculations awkward. Though this difficulty is presumably superable, the approach in this paper is more analytic and depends on the underlying hyperbolic geometry.

According to [W2], the Weil-Petersson Kähler two-form $\omega$ is a representative (actually, the harmonic representative) of $2 \pi^{2} \bar{\kappa}_{1}$ on $\overline{\mathcal{M}}$, and we now let $\kappa_{1}=$ $\omega /\left(2 \pi^{2}\right)$ denote this form on $\mathcal{M}$. Our main result (which is a weakening of the first of the conjectures above) is that the cycle $\left[W_{2}\right]$ of non-compact support on $\mathcal{M}$ is a constant multiple of the "compact Poincaré dual" of $\omega$ on $\mathcal{M}$, in the sense that for any compactly supported closed $(N-2)$-form $\eta$ on $\mathcal{M}$, we have $\int_{\mathcal{M}} \omega \wedge \eta=c \int_{\left[W_{2}\right]} \eta$, for some constant $c$ independent of $\eta$. In fact, we have

Theorem 0.1. $\kappa_{1}$ is the compact Poincaré dual of $\frac{1}{6}\left[W_{2}\right]$ on $\mathcal{M}$.

The proof is a computation using our explicit integration scheme [P4, §7], which we use here to integrate over various cycles in $\mathcal{M}$ rather than just over $\mathcal{M}$ itself as in [P3]. One ingredient of our approach is a simple lemma about any matrix-model, which allows us, in effect, to apply Stokes' theorem one cell at a time on $\mathcal{M}$. 
Using our coordinates on the Teichmüller space, the geometry leads naturally to exactly the Hain-MacPherson version of Rogers' function [HM] as well as to the Abel-Spence functional equation which Rogers' function satisfies.

This paper is organized as follows. Section 1 is dedicated to a quick review of the necessary decorated Teichmüller theory, and Section 2 presents our lemma about matrix-models. Various computations with our coordinates are performed in Section 3 in order to prove in Section 4 that Witten's putative cycles are indeed cycles. The computation of the compact Poincaré dual of the Weil-Petersson Kähler two-form is presented in Section 5. Section 6 is devoted to closing remarks, among which are various comments on the vagaries of compactifying $\mathcal{M}$ in the setting of this paper.

It is a pleasure to thank Ed Witten for many stimulating conversations and to acknowledge the intellectual debt to him of some of the work presented here. Jean-Louis Loday patiently explained connections between polylogarithms and K-theory and made many valuable comments. Not the least of these comments educated me about the Abel-Spence relation and thereby substantially shortened the proof given here (whose original sketch went through a painful partial derivation of the Abel-Spence relation). There were also stimulating and informative discussions with Osmo Pekonen and Dennis Sullivan. Let me finally thank Université Louis Pasteur and the Finnish Mathematical Society, especially Seppo Rickman, for support during Winter, 1990.

\section{BACKGROUND}

We begin by reviewing the decorated Teichmüller theory $[\mathrm{P} 1]-[\mathrm{P} 4]$ and refer the reader to $[\mathrm{P} 3, \S 3]$ for a more extensive review.

Let $F_{g}^{s}$ denote a fixed genus $g$ oriented topological surface with $s \geq 1$ punctures, where $2 g-2+s>0$. The (pure) mapping class group $M C_{g}^{s}$ of $F_{g}^{s}$ is the group of isotopy classes of orientation-preserving diffeomorphisms of $F_{g}^{s}$ which must pointwise fix the punctures (whereas we consider the impure mapping class group in [P1]-[P4]). The Teichmüller space $\mathcal{T}_{g}^{s}$ of $F_{g}^{s}$ is the collection of all marked hyperbolic structures (complete with constant curvature -1 ) modulo push-forward by diffeomorphisms isotopic to the identity, and the quotient $\mathcal{M}_{g}^{s}=\mathcal{T}_{g}^{s} / M G_{g}^{s}$ is the mcduli space of $F_{g}^{s}$, which is the object of central inter- 
est classically. We consider the fiber-space $\tilde{\mathcal{T}}_{g}^{s}$ over $\mathcal{T}_{g}^{s}$, where the fiber over a point of $\mathcal{T}_{g}^{s}$ is the collection of all $s$-tuples of horocycles, one horocycle about each puncture. $\tilde{\mathcal{T}}_{g}^{s}$ is called the decorated Teichmüller space of $F_{g}^{s}$ and plays a central role in [P1]-[P4]. We shall typically let $\Gamma \in \mathcal{T}_{g}^{s}$ denote a Fuchsian group underlying a point $\tilde{\Gamma} \in \tilde{\mathcal{T}}_{g}^{s}$, so the marking is understood. The action of $M C_{g}^{s}$ on $\mathcal{T}_{g}^{s}$ lifts to an action of $M C_{g}^{s}$ on $\tilde{\mathcal{T}}_{g}^{s}$ in the natural way.

Define an ideal triangulation $\Delta$ of $F_{g}^{s}$ to be (the isotopy class of) a collection of arcs disjointly embedded in $F_{g}^{s}$ running between (not necessarily distinct) punctures, so that each component of $F_{g}^{s}-\Delta$ is an ideal triangle in $F_{g}^{s}$ with its vertices among the punctures. (The isotopy class of) a subset $\Delta^{\prime} \subset \Delta$ of an ideal triangulation is called an ideal cell-decomposition or simply an "i.c.d." of $F_{g}^{s}$ provided that each complementary region of $\Delta^{\prime}$ in $F_{g}^{s}$ is an ideal polygon.

Suppose that $\tilde{\Gamma} \in \tilde{\mathcal{T}}_{g}^{s}$ and $\alpha$ is (the isotopy class of) an arc, as above, connecting punctures in $F_{g}^{s}$. Straighten $\alpha$ to a geodesic for $\Gamma$, choose a lift of $\alpha$ to a geodesic in the hyperbolic plane, and consider the ideal points to which this lift is asymptotic. The decoration on $\tilde{\Gamma}$ determines a pair of horocycles centered at these ideal points, and we define the lambda length of $\alpha$ and $\tilde{\Gamma}$ to be

$$
\lambda(\alpha ; \tilde{\Gamma})=\sqrt{2 e^{\delta}},
$$

where $\delta$ is the signed hyperbolic distance between these two horocycles. This quantity is clearly independent of our choices, and we have

Theorem A (Theorems 3.1 of [P1] and A.2 of [P3]). For any fixed ideal triangulation $\Delta$ of $F_{g}^{s}$, the function

$$
\begin{aligned}
\tilde{\mathcal{T}}_{g}^{s} & \rightarrow R_{+}^{\Delta} \\
\tilde{\Gamma} & \mapsto \Lambda, \text { where } \Lambda(\alpha)=\lambda(\alpha ; \tilde{\Gamma}) \text { for each } \alpha \in \Delta
\end{aligned}
$$

is a homeomorphism. Furthermore, the pull-back of the Weil-Petersson Kähler two-form on $\mathcal{T}_{g}^{s}$ is given by

$-2 \sum d \log a \wedge d \log b+d \log b \wedge d \log c+d \log c \wedge d \log a$,

where the sum is over the collection of triangles complementary to $\Delta$ in $F_{g}^{s}$, the lambda-lengths assigned to the frontier edges of this triangle are $a, b, c$ in 
this order as traversed in the counter-clockwise sense (in the orientation on $F_{g}^{s}$ ), and $\log$ denotes the natural logarithm.

Another ingredient of the decorated Teichmüller theory is the $M C_{g}^{s}$-invariant "cell- decomposition" of $\tilde{\mathcal{T}}_{g}^{s}$, whose attendant combinatorics is effectively captured by fatgraphs and the matrix-model. Recall that a fatgraph is a onedimensional CW-complex together with a cyclic ordering on the collection of half-edges about each vertex. One associates a surface to a fatgraph in the natural way, and a marking in the usual sense on this surface is called a marking on the underlying fatgraph. A cell in the decomposition of $\mathcal{T}_{g}^{s}$ corresponds to an i.c.d., and an i.c.d., in turn, corresponds via Poincaré duality on the surface to some marked fatgraph. We shall require both the i.c.d. and the fatgraph formalisms here, and therefore require several definitions:

Let $\Delta(G)$ be the i.c.d. corresponding to marked fatgraph $G$, let $G(\Delta)$ be the marked fatgraph corresponding to the i.c.d.

$\Delta$,

let $\mathcal{C}(\Delta)$ be the open cell in $\tilde{\mathcal{T}}_{g}^{s}$ corresponding to the i.c.d. $\Delta$, set $\mathcal{C}(G)=\mathcal{C}(\Delta(G))$, and

let $\overline{\mathcal{C}}(G)$ denote the closure in $\tilde{\mathcal{T}}_{g}^{s}$ of $\mathcal{C}(G)$.

If $\Delta$ is an ideal triangulation of $F_{g}^{s}$ and $e$ is an edge of $\Delta$, then the component of $\left(F_{g}^{s}-\Delta\right) \cup\{e\}$ containing $e$ is either a once-punctured monogon or a quadrilateral. In the latter case, we may replace $e$ with the other diagonal $e^{\prime}$ of the associated quadrilateral to produce a new ideal triangulation $\Delta^{\prime}=(\Delta-\{e\}) \cup\left\{e^{\prime}\right\}$. We say in this case that $\Delta^{\prime}$ arises from $\Delta$ by applying an elementary move along e. By definition, the corresponding fatgraphs are related by Whitehead equivalence.

The face relation in the "cell-decomposition" of $\tilde{\mathcal{T}}_{g}^{s}$ is induced by inclusion of i.c.d.'s in the natural way, and two top-dimensional cells share a common codimension-one face if and only if they differ by a elementary move. The dual of this relation gives a partial ordering on

$\mathcal{G}_{g}^{s}=\left\{\right.$ marked isomorphism classes of fatgraphs $G: \Delta(G)$ is an i.c.d. of $\left.F_{g}^{s}\right\}$, 
and we refer to the poset $\mathcal{G}_{g}^{s}$ as the fatgraph poset. Thus, $G \in \mathcal{G}_{g}^{s}$ is the name of a cell $\mathcal{C}(G)=\mathcal{C}(\Delta(G)) \subset \tilde{\mathcal{T}}_{g}^{s}$, and it is in this sense that we shall employ both formalisms below.

To analyze the structure of cells in the decomposition, fix some $G \in \mathcal{G}_{g}^{s}$. To each $\tilde{\Gamma} \in \mathcal{C}(G) \subset \tilde{\mathcal{T}}_{g}^{s}$ and each edge of $G$, there is associated its lambda length, that is, the lambda length of its dual ideal arc in $\Delta(G)$. There is furthermore associated another positive real quantity, called its "simplicial coordinate" (whose definition we shall recall when we need it in Lemma 3.2 below), and we have

Theorem B (Theorem 3.4.1 of [P3]). For any marked fatgraph $G$, let $\left(E_{i}\right)_{i=1}^{N}$ denote the tuple of simplicial coordinates associated to the respective edges $\left\{e_{i}\right\}_{i=1}^{N}$ of $G$. This tuple $\left(E_{i}\right)_{i=1}^{N}$ of positive real numbers gives global coordinates on $\mathcal{C}(G)$. Furthermore, if $G^{\prime}, G^{\prime \prime}$ are marked fatgraphs so that $\Delta\left(G^{\prime \prime}\right) \subset$ $\Delta\left(G^{\prime}\right)$, then $\mathcal{C}\left(G^{\prime \prime}\right) \subset \overline{\mathcal{C}}\left(G^{\prime}\right)$ is determined by the collection of equalities

$$
E_{i_{j}}=0, \text { for all } e_{i_{j}} \in \Delta\left(G^{\prime}\right)-\Delta\left(G^{\prime \prime}\right) .
$$

The decorated bundle $\tilde{\mathcal{T}}_{g}^{s} \rightarrow \mathcal{T}_{g}^{s}$ admits the canonical section where each horocycle in the decoration has hyperbolic length unity, and we may thus regard $\mathcal{T}_{g}^{s} \subset \tilde{\mathcal{T}}_{g}^{s}$. In fact, the "cell-decomposition" of $\tilde{\mathcal{T}}_{g}^{s}$ restricts to a "celldecomposition" $\mathcal{T}_{g}^{s}$. To see this, suppose that $G \in \mathcal{G}_{g}^{s}$. The fattening of $G$ determines a marked surface whose boundary components are in natural one-to-one correspondence with the ordered set of punctures of $F_{g}^{s}$, and each boundary component of this surface gives rise to a closed edge-path on $G$ in the natural way. For any $\tilde{\Gamma} \in \tilde{\mathcal{T}}_{g}^{s}$, let $\rho_{i}(\tilde{\Gamma})$ denote the sum (with unsigned multiplicity) of the simplicial coordinates of the edges traversed by this edgepath, for $i=1, \ldots, s$. By [P3, Corollary 3.4.3], $\rho_{i}(\tilde{\Gamma})$ is the hyperbolic length of the $i^{\text {th }}$ horocycle in the decorated conformal structure $\tilde{\Gamma}$. It follows that

$$
\mathcal{D}(G)=\mathcal{C}(G) \cap \mathcal{T}_{g}^{s} \subset \tilde{\mathcal{T}}_{g}^{s}
$$

is the affine slice $\left\{\rho_{i}=1: i=1, \ldots, s\right\}$ of the positive orthant in simplicial coordinates, so $\mathcal{D}(G)$ is in particular a cell, as was asserted. If $\Delta$ is an i.c.d. of $F_{g}^{s}$, then we shall also write simply $\mathcal{D}(\Delta)=\mathcal{D}(G(\Delta))$. 
The canonical section of $\tilde{\mathcal{T}}_{g}^{s} \rightarrow \mathcal{T}_{g}^{s}$ is everywhere transverse to $\partial \overline{\mathcal{C}}(G)$ by [P3, Lemma 3.2.2], and therefore

Corollary C. For any marked fatgraph $G \in \mathcal{G}_{g}^{s}$, let $\left(E_{i}\right)_{i=1}^{N}$ denote the tuple of simplicial coordinates associated to the respective edges $\left\{e_{i}\right\}_{i=1}^{N}$ of $G$. The affine slice of the simplicial coordinates on $\overline{\mathcal{C}}(G)$ determined by $\left\{\rho_{i}=1\right.$ : $i=1, \ldots, s\}$ gives global coordinates on the closure $\overline{\mathcal{D}}(G)$ of $\mathcal{D}(G)$ in $\mathcal{T}_{g}^{s}$. Furthermore, if $G^{\prime}, G^{\prime \prime}$ are marked fatgraphs so that $\Delta\left(G^{\prime \prime}\right) \subset \Delta\left(G^{\prime}\right)$, then $\mathcal{D}\left(G^{\prime \prime}\right) \subset \overline{\mathcal{D}}\left(G^{\prime}\right)$ is determined by the collection of equalities

$$
E_{i_{j}}=0, \text { for all } e_{i_{j}} \in \Delta\left(G^{\prime}\right)-\Delta\left(G^{\prime \prime}\right) .
$$

Turning finally to our integration scheme, given a fatgraph $G$, let Aut $(G)$ denote the unmarked fatgraph automorphism group of $G$, let $[G]$ denote the unmarked isomorphism class of $G$, and let \#Aut $[G]$ denote the cardinality of $\operatorname{Aut}(G)$ for any $G \in[G]$. Another invariant (which detects the "hyperellipticity") of $G$ is

$$
\epsilon[G]= \begin{cases}1, & \text { if there is a non-trivial element of } \operatorname{Aut}(G) \\ & \text { fixing each unoriented edge of } G ; \\ 0, & \text { otherwise }\end{cases}
$$

for any $G \in[G]$, and we say that $G$ or $[G]$ is hyperelliptic if $\epsilon[G]=1$. Observe that a hyperelliptic fatgraph $G$ must have either one or two vertices (cf. $[\mathrm{P} 3, \S 3.1]$ ); thus, there are no cells in $\mathcal{T}_{g}^{s}$ of codimension 0 , 1 , or 2 whose corresponding fatgraph is hyperelliptic unless $F_{g}^{s}=F_{1}^{1}, F_{0}^{4}, F_{1}^{2}$.

We shall only have occasion below to consider integrals of closed forms over certain cycles of non-compact support on $\mathcal{M}_{g}^{s}$. Namely, suppose that $Z$ is a $p$-cycle of non-compact support in the "cell-decomposition" of $\mathcal{T}_{g}^{s}$ which is invariant under the action of the mapping class group $M C_{g}^{s}$. Thus, the pushforward $[Z]=\phi_{*}(Z)$ is a well-defined $p$-cycle of non-compact support on $\mathcal{M}_{g}^{s}$, where $\phi: \mathcal{T} \rightarrow \mathcal{M}$ is the forgetful map, and it is cycles of this type which we shall consider in the sequel.

To establish notation for such cycles, enumerate the $M C_{g}^{s}$-orbits of $p$-cells in $\mathcal{T}_{g}^{s}$ as $\left\{\left[G_{k}\right]\right\}_{k=1}^{K}$, and choose an orientation on respective representative cells $\left\{\mathcal{D}\left(G_{k}\right)\right\}_{k=1}^{K}$ once and for all. If $[Z]$ is a $p$-cycle on $\mathcal{M}_{g}^{s}$ as above, then we write 
$[Z]=\sum_{k=1}^{K} n_{k}\left[G_{k}\right]$ if the restriction of the corresponding cycle $Z$ to $\mathcal{D}\left(G_{\ell}\right)$ is $n_{k} \in Q$ times the specified orientation on $\mathcal{D}\left(G_{k}\right)$.

Our integration scheme for this class of cycles on $\mathcal{M}_{g}^{s}$ is

Theorem D. [P4, Remark 1 after Theorem 2] Given a p-cycle $Z$ of noncompact support on $\mathcal{T}_{g}^{s}$ which is invariant under the action of $M C_{g}^{s}$, write $[Z]=\sum_{k=1}^{K} n_{k}\left[G_{k}\right]$, as above, which depends upon the finite collection of specifications of orientations on $\left\{\mathcal{D}\left(G_{\ell}\right)\right\}_{\ell=1}^{N}$. If $\xi$ is a closed $p$-form on $\mathcal{M}_{g}^{s}$, then

$$
\int_{[Z]} \xi=\sum_{k=1}^{K} \frac{2^{-\epsilon\left[G_{k}\right]}}{\# A u t\left[G_{k}\right]} n_{k} \int_{\mathcal{D}\left(G_{k}\right)} \phi^{*}(\xi)
$$

where $\phi^{*}(\xi)$ is the pull-back of $\xi$ to a p-form on $\mathcal{T}_{g}^{s}$, and each $\mathcal{D}\left(G_{k}\right)$ is given its specified orientation.

Remark 1.1. It is not difficult to prove that the projection $\mathcal{T}_{g}^{s} \rightarrow \mathcal{M}_{g}^{s}$ is an embedding on the closures of cells in the first barycentric subdivision of the "cell-decomposition" of $\mathcal{T}_{g}^{s}$. In fact, the second barycentric subdivision of the "cell-decomposition" actually descends to a "simplicial decomposition" of $\mathcal{M}_{g}^{s}$ (where the final quotation marks are explained as in the introduction); this point is discussed further in Section 6. One can easily extend the integration scheme above to one that allows arbitrary cycles of non-compact support on $\mathcal{M}_{g}^{s}$ in this first barycentric subdivision. In fact, this modified and more general integration scheme is already suggested by Lemma 2.1 below. On the other hand, we shall not need this more sophisticated integration scheme in this paper.

The Weil-Petersson Kähler two-form $\omega$ determines a volume-form $\omega^{3 g-3+s}$ (with non-compact support) on both the moduli space $\mathcal{M}_{g}^{s}$ and the Teichmüller space $\mathcal{T}_{g}^{s}$ of $F_{g}^{s}$. $\omega$ furthermore determines a canonical orientation on the decorated Teichmüller space $\tilde{\mathcal{T}}_{g}^{s}$, as follows. Let $\rho_{i}$ denote the hyperbolic length of the horocycle about the $i^{\text {th }}$ puncture, for $i=1, \ldots, s$. Since the punctures are ordered,

$$
\tilde{\omega}=\omega \wedge d \rho_{1} \wedge \cdots \wedge d \rho_{s}
$$

is a sensible definition, and $\tilde{\omega}$ is thus a canonical orientation on $\tilde{\mathcal{T}}_{g}^{s}$. 
If $G \in \mathcal{G}_{g}^{s}$ is a cubic fatgraph, then $\mathcal{D}(G)$ inherits an orientation from that of $\mathcal{T}_{g}^{s}$, and the integration scheme in Theorem $\mathrm{D}$ becomes

$$
\int_{\mathcal{M}_{g}^{s}} \xi=\sum_{[G]} \frac{2^{-\epsilon[G]}}{\# A u t[G]} \int_{\mathcal{D}(G)} \phi^{*}(\xi),
$$

with the understood orientation on each $\mathcal{D}(G)$, where the sum is over all unmarked isomorphism classes of cubic fatgraphs whose corresponding surface is homeomorphic to $F_{g}^{s}$.

\section{Fatgraphs and Stokes' Theorem}

If $G \in \mathcal{G}_{g}^{s}$, then recall from Section 1 that $[G]$ denotes the unmarked equivalence class and $\operatorname{Aut}(G)$ denotes the fatgraph symmetry group of $G$. We also let $v_{k}(G)$ denote the number of $k$-valent vertices of $G$ for any positive integer $k$ and define $s(G)=s \geq 1$ to be the number of punctures of the surface $F_{g}^{s}$ corresponding to $G$.

Notice first that the Euler characteristic of $G$ (and hence of $F_{g}^{s}$ ) is simply

$$
\chi(G)=\sum_{k}(k-2) v_{k}(G)
$$

and of course the pair $\chi=\chi(G), s=s(G)$ uniquely determines the corresponding surface $F_{\frac{1}{2}\{\chi+2-s\}}^{s}$. Furthermore, the codimension of $\mathcal{D}(G)$ in $\mathcal{T}_{g}^{s}$ (or, in other words, the codimension of $\mathcal{C}(G)$ in $\left.\tilde{\mathcal{T}}_{g}^{s}\right)$ is

$$
c(G)=\sum_{k}(k-3) v_{k}(G) .
$$

Notice that \#Aut, $v_{k}, s, \chi$, and $c$ are actually well-defined functions of the equivalence class $[G]$ of $G$, and write, for instance, $v_{k}[G]$ for the value of $v_{k}(G)$ for any $G \in[G]$.

Define

$$
\begin{aligned}
\left(*_{j}\right)_{g}^{s} & =\left\{G \in \mathcal{G}_{g}^{s}: c[G]=j\right\}, \\
{\left[*_{j}\right]_{g}^{s} } & =\left\{[G]: G \in\left(*_{j}\right)_{g}^{s}\right\},
\end{aligned}
$$

so that each $\left[*_{j}\right]_{g}^{s}$ is a finite set. In the sequel, we shall typically fix the genus $g$ and number $s$ of punctures and uniformly drop the subscript $g$ and superscript $s$. 
If $G \in\left(*_{j}\right)$, then define

$$
\begin{aligned}
& \partial_{j} G=\left\{H \in\left(*_{j+1}\right): \Delta(H) \subset \Delta(G)\right\} \subset\left(*_{j+1}\right), \\
& \delta_{j} G=\left\{H \in\left(*_{j-1}\right): \Delta(H) \supset \Delta(G)\right\} \subset\left(*_{j-1}\right) .
\end{aligned}
$$

Given a pair $(G, H)$ of elements of $\mathcal{G}$, where $H \in \partial_{j} G$ (or, in other words, $\left.G \in \delta_{j-1} H\right)$, let $[G, H]$ denote the unmarked isomorphism class of the pair $(G, H)$. Fixing $[G, H]$, a marking $G \in[G]$ uniquely determines a marking $H \in[H]$, and conversely.

For any $[G] \in\left[*_{j}\right]$, define

$$
\begin{aligned}
& \Phi[G]=\{[G, H]: \Delta(H) \subset \Delta(G)\} \\
& \Psi[G]=\{[H, G]: \Delta(H) \supset \Delta(G)\} .
\end{aligned}
$$

Noticing that the elements of $\Phi([G])$ and those of $\Psi([G])$ as well occur with certain well-defined multiplicities, we are led to define

$$
\begin{aligned}
& \phi_{[G]}\left[G_{1}\right]=\#\left\{H \in \partial_{j} G:[G, H]=\left[G, G_{1}\right]\right\} \\
& \psi_{\left[G_{1}\right]}[G]=\#\left\{H \in \delta_{j+1} G_{1}:\left[H, G_{1}\right]=\left[G, G_{1}\right]\right\},
\end{aligned}
$$

for any $\left[G_{1}\right] \in\left[*_{j+1}\right]$.

A basic result which allows us to apply Stokes' Theorem on the fatgraph complex is

Lemma 2.1. Fix any $g$ and $s$, and set $\left[*_{j}\right]=\left[*_{j}\right]_{g}^{s}$ for each $j$. To each pair $\left[G, G_{1}\right]$, assign a formal variable $X_{\left[G, G_{1}\right]}$, where we assume that $X_{\left[G, G_{1}\right]}$ vanishes if $\Delta\left(G_{1}\right) \not \subset \Delta(G)$. Then

$$
\begin{aligned}
\sum_{[G] \in\left[*_{j}\right]} \frac{1}{\# A u t[G]} \sum_{\left[G, G_{1}\right] \in \Phi[G]} \phi_{[G]}\left[G_{1}\right] X_{\left[G, G_{1}\right]} & =\sum_{\left[G_{1}\right] \in\left[*_{j+1}\right]} \frac{1}{\# A u t\left[G_{1}\right]} \sum_{\left[G, G_{1}\right] \in \Psi\left[G_{1}\right]} \psi_{\left[G_{1}\right]}[G] X_{\left[G, G_{1}\right]} .
\end{aligned}
$$

Proof. Fix some $[G, H] \in \Phi[G]$, and consider the coefficient of $X_{[G, H]}$ on either side of the equation in Lemma 2.1 .

Let the valence tuple of $[G]$ be $v_{3}=v_{3}[G], \ldots, v_{K}=v_{K}[G]$, and recall the enumeration of fatgraphs with this valence tuple given in the last step of Theorem 2.1 of $[\mathrm{P} 2]$. Thus, $[G]$ occurs in this enumeration $\rho[G]$ times, 
where $\rho[G] \# A u t[G]=\prod_{k=3}^{K} v_{k} ! k^{v_{k}}$. For each fatgraph $G^{\prime} \in[G]$ arising in this enumeration, there are $\phi_{[G]}[H]$ distinct edges which one may collapse to produce a fatgraph $H^{\prime}$ so that $\left(G^{\prime}, H^{\prime}\right) \in[G, H]$. Arguing as on p. 45 of [P2](using 1-labellings), we conclude that the coefficient of $X_{[G, H]}$ on the lefthand side of the formula in Lemma 2.1 is $1 / \# A u t[G, H]$, where $\operatorname{Aut}(G, H)$ is the automorphism group of the unmarked pair $(G, H)$.

In the same way, set $t_{3}=v_{3}[H], \ldots, t_{K}=v_{K}[H]$ and consider the enumeration of fatgraphs with valence tuple $t_{3}, \ldots, t_{K}$. Arguing exactly as in the previous paragraph, the coefficient of $X_{[G, H]}$ on the righthand side of the formula in Lemma 2.1 is again found to be $1 / \# A u t[G, H]$, completing the proof.

\section{Computations with Simplicial Coordinates}

It is more convenient here to consider the i.c.d. formalism as opposed to the fatgraph formalism. Recall that we have let $\Delta(G)$ denote the i.c.d. of $F=F_{g}^{s}$ corresponding to the marked isomorphism class of the fatgraph $G$ whose associated surface is homeomorphic to $F$. We may add geodesics to $\Delta(G)$ to produce an ideal triangulation $\Delta$ of $F$, and we linearly order the edges of $\Delta-\Delta(G)=\left\{e_{i}\right\}_{i=1}^{M}$. To each $e_{i} \in \Delta$ is associated its simplicial coordinate $E_{i}$, and it follows from Corollary $\mathrm{C}$ above that $d E_{1} \wedge \cdots \wedge d E_{M}$ is a co-normal vector to $\mathcal{D}(G)$ in $\mathcal{T}$. Insofar as $\mathcal{T}$ comes equipped with its orientation derived from the two-form $\omega$ (as in Section 1), we conclude

Lemma 3.1. Given $G \in \mathcal{G}$, choose an ideal triangulation $\Delta$ containing $\Delta(G)$, and choose a linear ordering on $\Delta-\Delta(G)$. Then there is a canonically determined orientation on $\mathcal{D}(G)$.

This is the way in which we shall typically specify an orientation on a cell of $\mathcal{T}$ in the sequel. Of course, in the special case that $G \in\left(*_{1}\right)$, the unique non-triangular region complementary to $\Delta(G)$ in $F$ is an ideal quadrilateral, and an orientation on $\mathcal{D}(G)$ is thus determined by a choice of diagonal of this quadrilateral.

To understand how these orientations transform, suppose that $\Delta, \Delta^{\prime}$ are ideal triangulations of $F$, where $\Delta^{\prime}$ arises from $\Delta$ by applying a single elemen- 


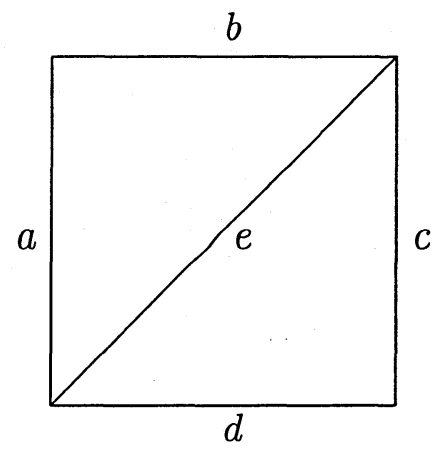

$\Delta$

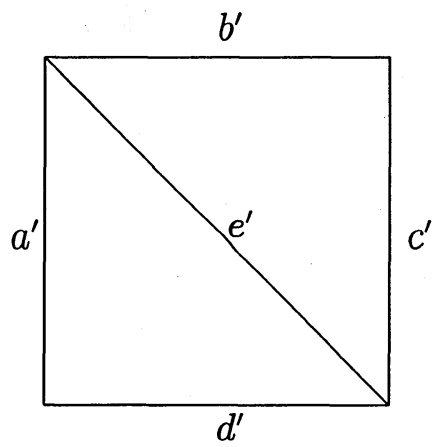

$\Delta^{\prime}$

\section{Figure 1}

tary move, and adopt the notation of Figure 1, where the lower-case symbol next to an edge indicates its corresponding lambda length, and the edge labelled $x^{\prime}$ of $\Delta^{\prime}$ corresponds to the edge labelled $x$ of $\Delta$. At the same time, we shall let the corresponding upper-case letter $X$ and $X^{\prime}$ denote the simplicial coordinate of the respective edges. As was promised in the introduction, the formula

$$
E=\frac{a b\left(c^{2}+d^{2}-e^{2}\right)+c d\left(a^{2}+b^{2}-e^{2}\right)}{a b c d e}
$$

defines the simplicial coordinate $E$ on the edge labelled " $e$ " in terms of the lambda lengths $a, b, c, d, e$ of nearby edges. The analogous formula is used to define the simplicial coordinate of any edge.

Recall (see [P1] or [P3]) that up to the action of the Möbius group, there is a unique lift of the vertices of the quadrilateral illustrated in Figure 1 to points in the light-cone in Minkowski space so that the lambda length of each edge is the square-root of the negative of the corresponding Minkowski inner-product. The closed convex hull of these points is a tetrahedron, and the signed volume $V$ of this tetrahedron is related to the simplicial coordinate $E$ on the edge labelled $e$ by the formula $V=a b c d e E$. This gives a geometric interpretation to the simplicial coordinate.

Lemma 3.2. Given ideal triangulations $\Delta, \Delta^{\prime}$, where $\Delta^{\prime}$ arises from $\Delta$ by 
applying an elementary move along some edge, adopt notation as in Figure 1. Letting $X$ denote the simplicial coordinate of an arbitrary edge of $\Delta$, we have

$$
\begin{aligned}
X^{\prime} & =X, \quad \text { if } X \notin\{A, B, C, D, E\} \\
E^{\prime} & =-E, \\
B^{\prime}+C^{\prime} & =B+C+E, \\
A^{\prime}+B^{\prime}+E^{\prime} & =A+B \\
A^{\prime}+D^{\prime} & =A+D+E, \\
C^{\prime}+D^{\prime}+E^{\prime} & =C+D .
\end{aligned}
$$

The proof follows immediately from the definition of simplicial coordinates as the reader can easily verify. Actually, we shall only require the second formula $E^{\prime}=-E$ in the sequel but include these other linear formulas here for completeness.

Proposition 3.3. Continuing with the notation developed above for Figure 1, we have

(i) $\operatorname{det} \frac{\partial\left(E^{\prime}, C^{\prime}\right)}{\partial(E, C)} \leq 0$ provided $C=0$,

(ii) $\operatorname{det} \frac{\partial\left(A^{\prime}, E^{\prime}, C^{\prime}\right)}{\partial(A, E, C)} \leq 0$ provided $A=C=0$,

where det denotes the determinant, and $\frac{\partial\left(X_{1}^{\prime}, \ldots, X_{n}^{\prime}\right)}{\partial\left(X_{1}, \ldots, X_{n}\right)}$ denotes the Jacobian of $\left(X_{1}^{\prime}, \ldots, X_{n}^{\prime}\right)$ with respect to $\left(X_{1}, \ldots, X_{n}\right)$.

Proof. We begin with part (i), and observe that

$$
\frac{\partial\left(E^{\prime}, C^{\prime}\right)}{\partial(E, C)}=\left(\begin{array}{cc}
-1 & 0 \\
\frac{\partial C^{\prime}}{\partial E} & \frac{\partial C^{\prime}}{\partial C}
\end{array}\right)
$$

by the second part of Lemma 3.2. Thus, we must show that $\frac{\partial C^{\prime}}{\partial C} \geq 0$ if $C=0$, and this is obvious from the geometric interpretation of simplicial coordinates: Increasing the simplicial coordinate $C$ from zero alters the configuration of points in the light-cone in such a way that the corresponding tetrahedron has positive volume, and this in turn implies that $C^{\prime}$ must have increased from zero as well. 
Turning to part (ii), we have

$$
\frac{\partial\left(A^{\prime}, E^{\prime}, C^{\prime}\right)}{\partial(A, E, C)}=\left(\begin{array}{ccc}
\frac{\partial A^{\prime}}{\partial A} & \frac{\partial A^{\prime}}{\partial E} & \frac{\partial A^{\prime}}{\partial C} \\
0 & -1 & 0 \\
\frac{\partial C^{\prime}}{\partial A} & \frac{\partial C^{\prime}}{\partial E} & \frac{\partial C^{\prime}}{\partial C}
\end{array}\right)
$$

by the second part of Lemma 3.2, and so

$$
\operatorname{det} \frac{\partial\left(A^{\prime}, E^{\prime}, C^{\prime}\right)}{\partial(A, E, C)}=-\operatorname{det}\left(\begin{array}{ll}
\frac{\partial A^{\prime}}{\partial A} & \frac{\partial A^{\prime}}{\partial C} \\
\frac{\partial C^{\prime}}{\partial A} & \frac{\partial C^{\prime}}{\partial C}
\end{array}\right) .
$$

Again resorting to the geometric interpretation of simplicial coordinates, it is obvious that $\frac{\partial A^{\prime}}{\partial C}=0=\frac{\partial C^{\prime}}{\partial A}$ since $A=C=0$, while $\frac{\partial A^{\prime}}{\partial A} \geq 0, \frac{\partial C^{\prime}}{\partial C} \geq 0$ as before.

\section{Witten's CyCles}

Consider the collection

$$
W_{j}=\left\{G \in\left(*_{j}\right): v_{j+3}(G)=1\right\}
$$

of cells in $\mathcal{G}=\mathcal{G}_{g}^{s}$. We shall see that each cell $\mathcal{D}(G)$, for $G \in W_{2 i}$, admits a canonical orientation, and with these orientations, $W_{2 i}$ becomes a cycle with non-compact support on $\mathcal{T}$. In fact, these cycles are obviously equivariant for the action of the mapping class group on $\mathcal{T}$, and $W_{2 i}$ therefore descends to a cycle $\left[W_{2 i}\right]$ of non-compact support on $\mathcal{M}$.

To understand the orientation on a cell in $W_{j}$, consider the corresponding i.c.d. $\Delta(G)$. By definition, there is exactly one non-triangular complementary region of $\Delta(G)$ in $F$, and this region $P$ is an ideal $(j+3)$-gon. Choosing a vertex $v$ of $P$, there is a canonical ideal triangulation $\Delta_{v}$ of $P$ defined by the condition that each edge of $\Delta_{v}$ has $v$ as an ideal endpoint. The orientation on $F$ determines a linear ordering $\left\{e_{k}\right\}_{k=1}^{j}$ on $\Delta_{v}$, as in Figure 2. There is thus a canonical orientation on $\mathcal{D}(G)$ determined (as in Lemma 3.1) by each choice of vertex $v$ of $P$, namely that determined by the co-normal vector $d E_{1} \wedge \cdots \wedge d E_{j}$, where $E_{k}$ denotes the simplicial coordinate on the edge $e_{k}$, for $k=1, \ldots, j$. Let $\Omega_{v}$ denote the orientation so determined on $\mathcal{D}(G)$ for each $G \in W_{j}, j \geq 1$, and choice of vertex $v$. 


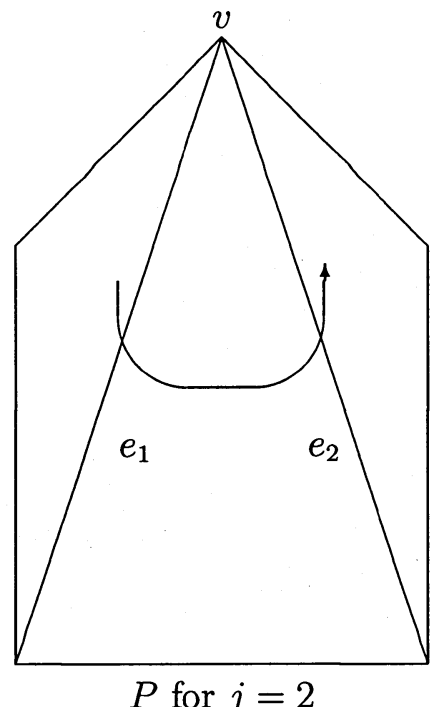

FiguRE 2

Lemma 4.1. Let $P$ be the $(j+3)$-gon complementary to $\Delta(G)$ in $F$, where $G \in W_{j}$. Suppose that $v_{1}$ and $v_{2}$ are consecutive vertices of $P$, and let them determine the respective orientations $\Omega_{1}=\Omega_{v_{1}}$ and $\Omega_{2}=\Omega_{v_{2}}$, as above, on $\mathcal{D}(G)$. Then $\Omega_{1}=\Omega_{2}$ if and only if $j$ is even. That is,

(i) If $j \equiv 0(2)$, then $\Omega_{1}=+\Omega_{2}$.

(ii) If $j \equiv 1(2)$, then $\Omega_{1}=-\Omega_{2}$.

Proof. Suppose that $v_{1}, v_{2}$ are consecutive vertices of $P$ occuring in this order in a counter-clockwise traversal of the frontier of $P$, and enumerate $\Delta_{v_{1}}=$ $\left\{e_{k}\right\}_{k=1}^{j}$, where the ordering on subscripts as before reflects the canonical ordering induced by the orientation of $F$. Set $\Delta_{0}=\Delta_{v_{1}}$, and recursively define $\Delta_{\ell}$ to be the ideal triangulation derived from $\Delta_{\ell-1}$ by an elementary move along $e_{\ell}$, for $\ell=1, \ldots, j$. The edge $e_{\ell}$ of $\Delta_{\ell-1}$ gives rise to an edge $e_{\ell}^{\prime}$ of $\Delta_{\ell}$ in the natural way; see Figure 3.

As in Lemma 3.1, the specification of co-normal vector

$$
d E_{1}^{\prime} \wedge \cdots \wedge d E_{\ell}^{\prime} \wedge d E_{\ell+1} \wedge \cdots \wedge d E_{j}
$$

determines an orientation on $\mathcal{D}\left(\Delta_{\ell}\right)$, where $E_{k}$ (and $E_{k}^{\prime}$, respectively) denotes 

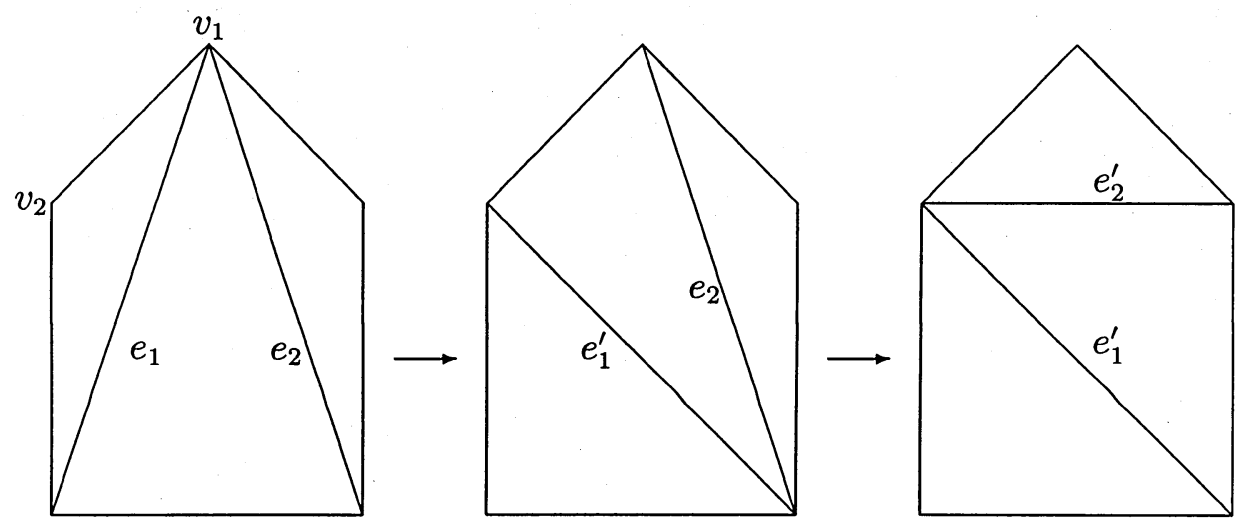

FiguRE 3

the simplicial coordinate of $e_{k}$ (and $e_{k}^{\prime}$ ). According to Proposition 3.3(i), the first and last elementary moves in the sequence $\Delta_{0} \rightarrow \Delta_{1} \rightarrow \cdots \rightarrow \Delta_{j}$ each preserve these orientations, while each of the intermediary $(j-2) \equiv j(2)$ moves reverses orientation by Proposition 3.3(ii). Thus, the composition of moves is orientation-preserving if and only if $j$ is even, as was asserted.

Each cell in $W_{2 i}$ therefore admits a canonical orientation, and we henceforth let $W_{2 i}$ represent the corresponding chain on $\mathcal{T}$ of non-compact support. The main result of this section is

Theorem 4.2. For each $i$, the chain $W_{2 i}$ is a cycle of non-compact support on $\mathcal{T}$. Furthermore, each such cycle descends to a rational cycle of non-compact support on $\mathcal{M}$.

Proof. We first show that $W_{2 i}$ is a cycle, and suppose that $G \in W_{2 i}$ and $G_{1} \in\left(*_{2 i+1}\right)$ with $\mathcal{D}\left(G_{1}\right) \subset \overline{\mathcal{D}}(G)$. There are the following two possibilities for $\Delta\left(G_{1}\right)$ : Either it has one complementary $(2 i+4)$-gon in $F$, or it has a complementary $(2 i+3)$-gon in addition to a complementary quadrilateral in $F$.

In the first case, Lemma 4.1(ii) (together with symmetry) shows that the coefficient of $\mathcal{D}\left(G_{1}\right)$ in the boundary of $W_{2 i}$ must vanish. In the second case, there are exactly two cells in $\mathcal{G}$ with $\mathcal{D}(G)$ in their boundary, and these contribute 
with opposite signs by the second part of Lemma 3.2. We again conclude that the coefficient of $\mathcal{D}\left(G_{1}\right)$ must vanish, so the chain $W_{2 i}$ is indeed a cycle.

Since the action of the mapping class group preserves the "cell-decomposition" and any mapping class is induced by an orientation-preserving homeomorphism of $F$, it follows that $W_{2 i}$ descends to a rational cycle of non-compact support on the moduli space, as was asserted.

Let $\left[W_{2 i}\right]$ denote the cycle of non-compact support on $\mathcal{M}$ corresponding to $W_{2 i}$ by the previous theorem.

\section{The Dual of the Weil-Petersson Kähler Two-Form}

This entire section is dedicated to a proof of

Theorem 5.1. Consider a surface $F=F_{g}^{s}$ and let $\omega$ denote the Weil-Petersson Kähler two-form on the moduli space $\mathcal{M}$ of $F$. For any closed $(6 g-8+2 s)$-form $\eta$ of compact support on $\mathcal{M}$, we have

$$
\int_{\mathcal{M}} \omega \wedge \eta=\frac{\pi^{2}}{3} \int_{\left[W_{2}\right]} \eta
$$

Our proof is computational and relies on the integration scheme. Hyperelliptic fatgraphs occur in codimension 0,1 , or 2 only for the surfaces $F_{1}^{1}, F_{0}^{4}, F_{1}^{2}$, and we assume to begin with that our surface $F_{g}^{s}$ is not among these exceptional cases. As before, we shall also typically drop the subscript $g$ and superscript $s$.

For this section only, we establish the conventions that for any real quantity $x$ defined on $\tilde{\mathcal{T}}$, we set

$$
\begin{aligned}
& \bar{x}=\log x, \\
& \tilde{x}=d \log x .
\end{aligned}
$$

Thus, for any cubic fatgraph $G$ the Weil-Petersson Kähler two-form on the corresponding moduli space is given by

$$
\omega=-2 \sum \tilde{a} \wedge \tilde{b}+\tilde{b} \wedge \tilde{c}+\tilde{c} \wedge \tilde{a},
$$


where the sum is over all vertices $v$ of $G$, and we assume that lambda lengths of the half-edges of $G$ incident on $v$ are $a, b, c$ in this cyclic order (as determined by the fattening).

Suppose now that $\eta$ is a closed $(6 g-8+2 s)$-form of compact support on $\mathcal{M}$. Our main task here is to compute $\int_{\mathcal{M}} \omega \wedge \eta$. According to the usual integration scheme applied to a volume-form on $\mathcal{M}$, we have

$$
\int_{\mathcal{M}} \omega \wedge \eta=\sum_{[G] \in\left[*_{0}\right]} \frac{1}{\# A u t[G]} \int_{\mathcal{D}(G)} \omega \wedge \eta
$$

where each $\mathcal{D}(G)$ is given the orientation induced by $\omega$ (as in $\S 1$ ).

For each $G \in \mathcal{G}$ with $[G] \in\left[*_{0}\right]$, define

$$
\xi_{G}=\sum[\bar{a} \tilde{b}+\bar{b} \tilde{c}+\bar{c} \tilde{a}-\{\bar{b} \tilde{a}+\bar{c} \tilde{b}+\bar{a} \tilde{c}\}],
$$

where the sum is again over all vertices of $G$ and the notation is as above for $\omega$. Notice that $\xi_{G}$ is not invariant under Ptolemy transformations (see [P1] and [P4]), but $\xi_{G}$ is clearly "natural" in the sense that for any $f \in \operatorname{Aut}(G)$, we have $f^{*}\left(\xi_{G}\right)=\xi_{G}$.

Of course, $\left.\omega\right|_{\mathcal{D}(G)}=-\left.d \xi_{G}\right|_{\mathcal{D}(G)}$, so by Stokes' Theorem (since $\eta$ is compactly supported)

$$
\int_{\mathcal{D}(G)} \omega \wedge \eta=-\int_{\partial \overline{\mathcal{D}}(G)} \xi_{G} \wedge \eta
$$

Notice that for each edge $e$ of $\Delta(G), \Delta(G)-\{e\}$ is an i.c.d. of $F$ if $F$ is oncepunctured, whereas in the multiply-punctured case, there are codimension-one faces of $\mathcal{D}(G)$ lying "at infinity" (and, of course, $\eta$ vanishes on these faces).

For any $G_{1} \in \mathcal{G}$ so that $\left[G, G_{1}\right] \in \Phi([G])$, let $\mathcal{D}_{\left[G, G_{1}\right]}\left(G_{1}\right)$ be the orientation on $\mathcal{D}\left(G_{1}\right)$ induced as a face of $\overline{\mathcal{D}}(G)$ with its specified orientation. Thus,

$$
\begin{aligned}
\int_{\mathcal{M}} \omega \wedge \eta & =\sum_{[G] \in\left[*_{0}\right]} \frac{-1}{\# A u t[G]} \int_{\partial \overline{\mathcal{D}}(G)} \xi_{G} \wedge \eta \\
& =\sum_{[G] \in\left[*_{0}\right]} \frac{-1}{\# A u t[G]} \sum_{\left[G, G_{1}\right] \in \Phi[G]} \phi_{[G]}\left[G_{1}\right] \int_{\mathcal{D}_{\left[G, G_{1}\right]}\left(G_{1}\right)} \xi_{G} \wedge \eta \\
& =\sum_{\left[G_{1}\right] \in\left[*_{1}\right]} \frac{-1}{\# A u t\left[G_{1}\right]} \sum_{\left[G, G_{1}\right] \in \Psi\left[G_{1}\right]} \psi_{\left[G_{1}\right]}[G] \int_{\mathcal{D}_{\left[G, G_{1}\right]}\left(G_{1}\right)} \xi_{G} \wedge \eta,
\end{aligned}
$$

where we have used naturality of $\xi_{G}$ and the hypothesis that $\eta$ is compactly supported in order to apply Lemma 2.1 in the last equality. 

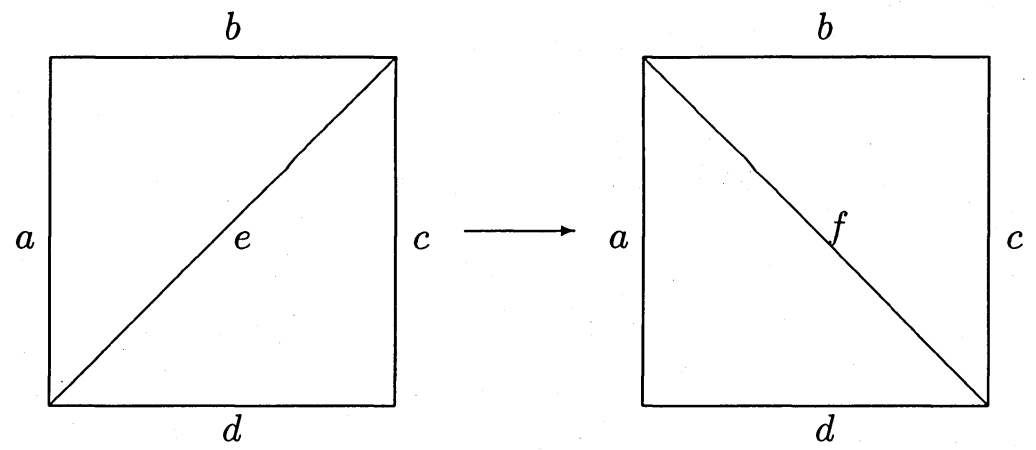

FIGURE 4

Choose now an orientation on $\mathcal{D}\left(G_{1}\right)$ for each $G_{1} \in\left(*_{1}\right)$ (that is, choose a diagonal $e$ of the quadrilateral complementary to $\Delta\left(G_{1}\right)$ in $F$ ), and let the other possible diagonal be denoted $f$, as in Figure 4. Suppose that $G=$ $G\left(\{e\} \cup \Delta\left(G_{1}\right)\right), H=G\left(\{f\} \cup \Delta\left(G_{1}\right)\right)$, and define

$$
\begin{aligned}
\xi_{G_{1}} & =\xi_{G}-\xi_{H} \\
& =\overline{a\left(\frac{b d}{e f}\right)}+\tilde{b} \overline{\left(\frac{e f}{a c}\right)}+\overline{\tilde{c}\left(\frac{b d}{e f}\right)}+\tilde{\tilde{d}} \overline{\left(\frac{e f}{a c}\right)}+\tilde{e} \overline{\left(\frac{a c}{b d}\right)}+\overline{f\left(\frac{a c}{b d}\right)}
\end{aligned}
$$

Recall that $e f=a c+b d$ by Ptolemy's Theorem [P3, Lemma 3.3.3], whence

$$
\tilde{e}+\tilde{f}=\frac{a c(\tilde{a}+\tilde{c})+b d(\tilde{b}+\tilde{d})}{a c+b d}
$$

so that, in fact,

$$
\xi_{G_{1}}=(\tilde{a}+\tilde{c}) \overline{\left(\frac{b d}{a c+b d}\right)}+(\tilde{b}+\tilde{d}) \overline{\left(\frac{a c+b d}{a c}\right)}+\overline{\left(\frac{a c}{b d}\right)} \frac{a c(\tilde{a}+\tilde{c})+b d(\tilde{b}+\tilde{d})}{a c+b d} .
$$

Notice that this previous expression for $\xi_{G_{1}}$ is independent of $e$ and $f$, is closed, and changes sign under the cyclic permutation of $a, b, c, d$.

An elementary calculation shows that $\xi_{G_{1}}=d \zeta_{G_{1}}$, where

$$
\zeta_{G_{1}}=2 \varphi\left(\frac{b d}{a c+b d}\right)
$$


and $\varphi(x)=\frac{1}{2}\left\{L i_{2}(x)-L i_{2}(1-x)\right\}$ is the Hain-MacPherson [HM] version of Rogers' function ( $L i_{2}(x)=\sum_{n \geq 1} n^{-2} x^{n}$ denoting Euler's dilogarithm). Notice that $\zeta_{G_{1}}$ changes sign under a change of orientation on $\mathcal{D}\left(G_{1}\right)$. (The choice of integration constant here is dictated by our application of Lemma 2.1 below.)

Invoking Stokes' Theorem again, we find

$$
\begin{aligned}
\int_{\mathcal{M}} \omega \wedge \eta & =\sum_{\left[G_{1}\right] \in\left[*_{1}\right]} \frac{-1}{\# A u t\left[G_{1}\right]} \int_{\partial \overline{\mathcal{D}}\left(G_{1}\right)} \zeta_{G_{1}} \wedge \eta \\
& =\sum_{\left[G_{1}\right] \in\left[*_{1}\right]} \frac{-1}{\# A u t\left[G_{1}\right]} \sum_{\left[G_{1}, G_{2}\right] \in \Phi\left[G_{1}\right]} \phi_{\left[G_{1}\right]}\left[G_{2}\right] \int_{\mathcal{D}_{\left[G_{1}, G_{2}\right]}\left(G_{2}\right)} \zeta_{G_{1}} \wedge \eta .
\end{aligned}
$$

In order to proceed and apply Lemma 2.1 , we must address the invariance of the integrals in the previous equation. There are two kinds of pairs $\left(G_{1}, G_{2}\right)$ here:

case (a): $\Delta\left(G_{2}\right)$ has two complementary quadrilaterals, and the edge in $\Delta\left(G_{1}\right)-\Delta\left(G_{2}\right)$ triangulates one of these quadrialterals.

case (b): $\Delta\left(G_{2}\right)$ has exactly one complementary pentagon, and the edge in $\Delta\left(G_{1}\right)-\Delta\left(G_{2}\right)$ decomposes this pentagon into a triangle and a quadrilateral.

Pairs of the latter type obviously admit no non-trivial automorphisms. Consider a pair $\left(G_{1}, G_{2}\right)$ of the former type. An automorphism $\iota$ of the pair must fix the quadrilateral complementary to $\Delta\left(G_{1}\right)$, and we choose a diagonal $e$ of this quadrilateral. $\iota$ may preserve $e$ setwise (in which case the orientation on $\mathcal{D}_{\left[G_{1}, G_{2}\right]}\left(G_{2}\right)$ and $\zeta_{G_{1}}$ are both unchanged), or it may not (in which case the orientation on $\mathcal{D}_{\left[G_{1}, G_{2}\right]}\left(G_{2}\right)$ and $\zeta_{G_{1}}$ both change sign).

Thus, we may apply Lemma 2.1 (again using that $\eta$ is compactly supported), to the effect that

$$
\int_{\mathcal{M}} \omega \wedge \eta=\sum_{\left[G_{2}\right] \in\left[*_{2}\right]} \frac{-1}{\# A u t\left[G_{2}\right]} \sum_{\left[G_{1}, G_{2}\right] \in \Psi\left[G_{2}\right]} \psi_{\left[G_{2}\right]}\left[G_{1}\right] \int_{\mathcal{D}_{\left[G_{1}, G_{2}\right]}\left(G_{2}\right)} \zeta_{G_{1}} \wedge \eta
$$

The terms in the previous sum are of two types as in case (a) and (b) above. In the former case, $\Delta\left(G_{2}\right)$ has a pair of complementary quadrilaterals, and there are four fatgraphs $G_{i}, G_{i i}, G_{i i i}, G_{i v}$ whose corresponding cells are incident on that corresponding to $G_{2}$, as illustrated in Figure 5. Owing to the change of orientation, the contribution from $\left[G_{i}, G_{2}\right]$ annihilates the contribution from 

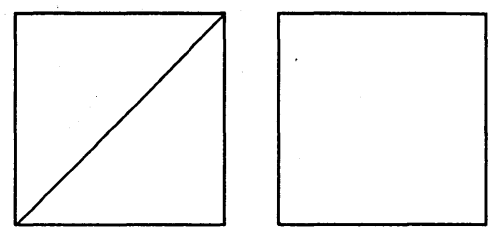

$\Delta\left(G_{i}\right)$
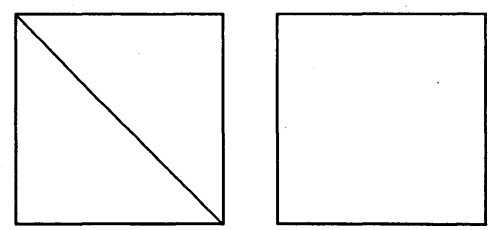

$\Delta\left(G_{i i}\right)$
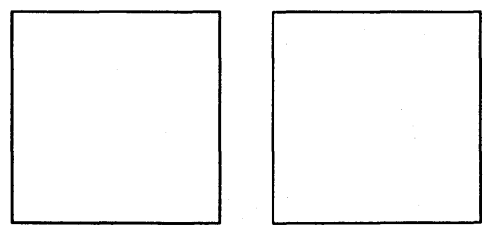

$\Delta\left(G_{2}\right)$
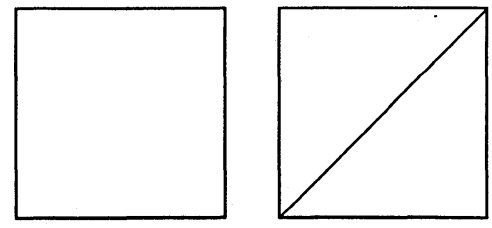

$\Delta\left(G_{i i i}\right)$
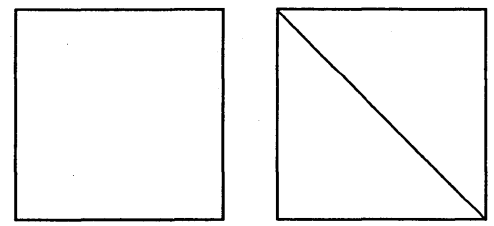

$\Delta\left(G_{i v}\right)$

FiguRe 5

$\left[G_{i i i}, G_{2}\right]$, and the contribution from $\left[G_{i i}, G_{2}\right]$ annihilates the contribution from $\left[G_{i v}, G_{2}\right]$.

In the remaining case (b), $\Delta\left(G_{2}\right)$ has a complementary pentagon $P$, and we adopt the notation for the frontier edges and vertices of $P$ as in Figure 6. (The subscripts will be taken modulo 4 here, so $v_{5}=v_{1}$ for instance.) There is a natural correspondence between the frontier edges and the various possible diagonals of $P$ (as is also indicated in the figure), and we adopt the notation of Figure 6 for these diagonals. We also illustrate in Figure 6 the various i.c.d.'s $\Delta^{m}$ which contain $\Delta\left(G_{2}\right)$ and let $G^{m}=G\left(\Delta^{m}\right)$, for $m=0, \ldots, 4$. To specify an orientation on $\mathcal{D}\left(G^{m}\right)$, we specify that the edge $e_{m}^{*}$ is the chosen diagonal 
of $\Delta^{m}$ so that the canonical orientation on $\mathcal{D}\left(G_{2}\right)$ (as in the previous section) actually agrees with the orientation $\mathcal{D}_{\left[G^{m}, G_{2}\right]}\left(G_{2}\right)$ for each $m=0, \ldots, 4$.

Thus, the contribution to $\int_{\mathcal{M}} \omega \wedge \eta$ from $\left[G_{2}\right] \in\left[*_{2}\right]$ is $\beta\left[G_{2}\right] \frac{1}{\# A u t\left[G_{2}\right]} \int_{\mathcal{D}\left(G_{2}\right)} \eta$, where

$$
\begin{aligned}
\beta\left[G_{2}\right] & =-2 \sum_{i=0}^{4} \varphi\left(\frac{e_{i} e_{i}^{*}}{e_{i} e_{i}^{*}+e_{i-1} e_{i+1}}\right) \\
& =-2 \sum_{i=0}^{4} \varphi\left(\frac{1}{1+\frac{e_{i-1} e_{i+1}}{e_{i} e_{i}^{*}}}\right) \\
& =-2 \sum_{i=0}^{4} \varphi\left(\frac{1}{1-\chi_{i}}\right)
\end{aligned}
$$

with

$$
\begin{aligned}
\chi_{i} & =C R\left(v_{i+2}, v_{i+1}, v_{i} ; v_{i-1}\right) \\
& =-\frac{e_{i-1} e_{i+1}}{e_{i} e_{i}^{*}}
\end{aligned}
$$

by [P3, Remark after Lemma A.1], where $C R(a, b, c ; z)$ is the value of $z$ under the Möbius transformation taking $a, b, c$, respectively, to $0,1, \infty$.

Actually, we shall employ the notation of [Du] for cross-ratios and set

$$
\{a: b: c: d\}=\frac{a-c}{b-a} \cdot \frac{c-a}{c-d}
$$

so $\frac{1}{1-\chi_{i}}=\left\{v_{i-1}: v_{i+2}: v_{i}: v_{i+1}\right\}$, for each $i=0, \ldots, 4$, and we define

$$
\begin{aligned}
& \lambda_{0}=\frac{1}{1-\chi_{2}}=\left\{v_{1}: v_{4}: v_{2}: v_{3}\right\}, \\
& \lambda_{1}=\frac{1}{1-\chi_{3}}=\left\{v_{2}: v_{0}: v_{3}: v_{4}\right\}, \\
& \lambda_{2}=\frac{1}{1-\chi_{4}}=\left\{v_{3}: v_{1}: v_{4}: v_{0}\right\}, \\
& \lambda_{3}=\frac{1}{1-\chi_{0}}=\left\{v_{4}: v_{2}: v_{0}: v_{1}\right\}, \\
& \lambda_{4}=\frac{1}{1-\chi_{1}}=\left\{v_{0}: v_{3}: v_{1}: v_{2}\right\}
\end{aligned}
$$

Lemma 5.2. Set $\lambda=\{a: b: c: d\}$. Then we have

$$
\{b: c: d: a\}=\frac{\lambda}{\lambda-1},\{a: c: b: d\}=1-\lambda, \text { and }\{a: b: d: c\}=\frac{1}{\lambda} .
$$



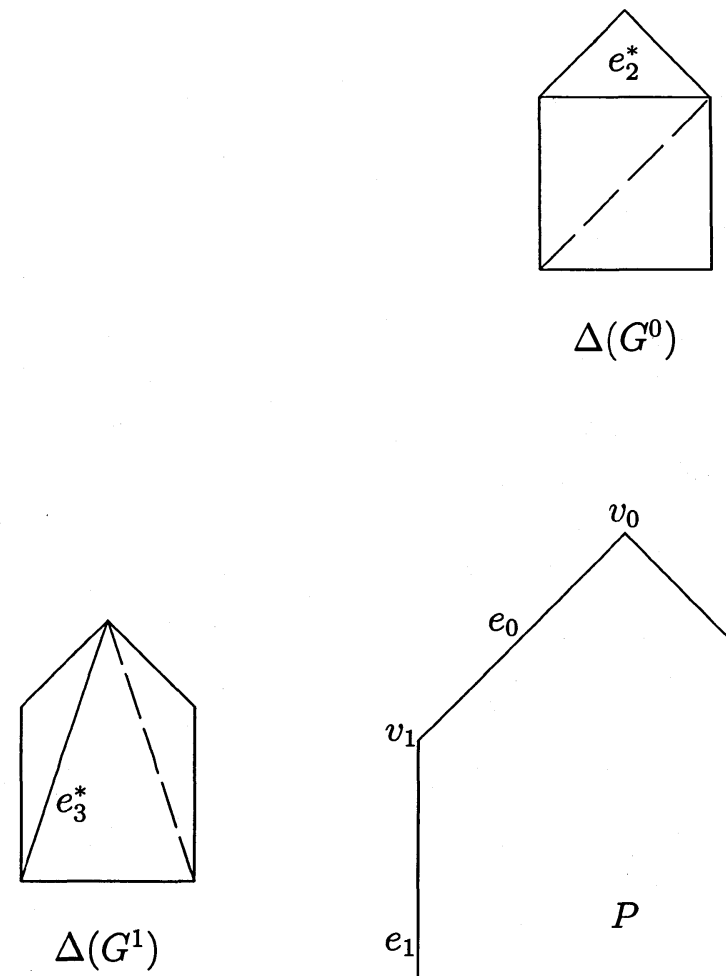

$$
\Delta\left(G^{0}\right)
$$
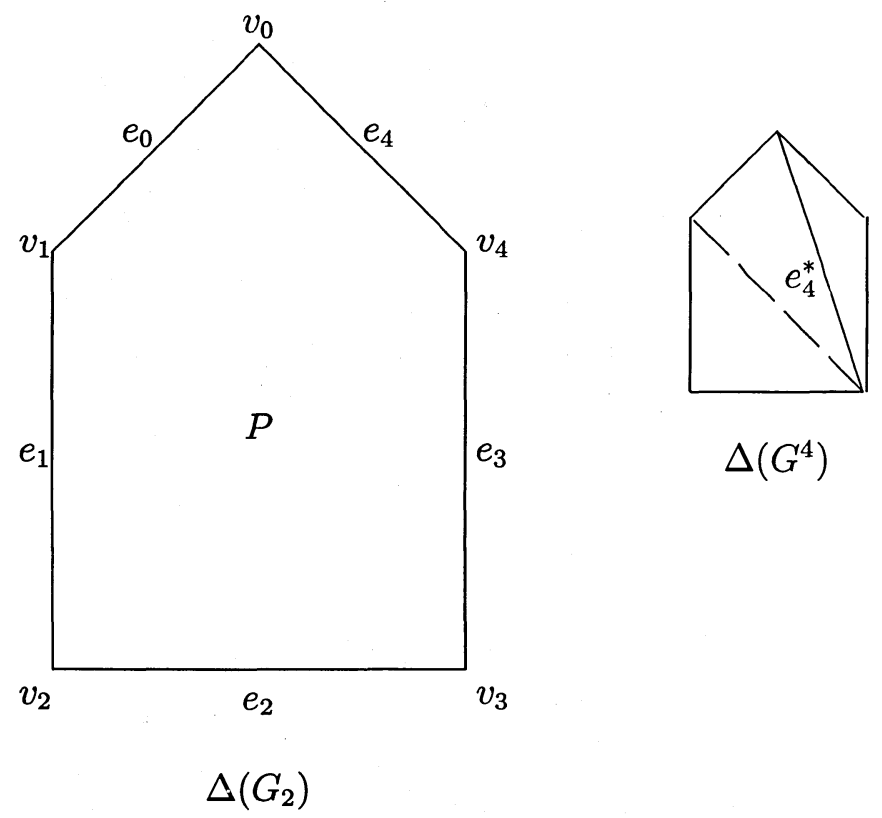

$$
\Delta\left(G^{4}\right)
$$
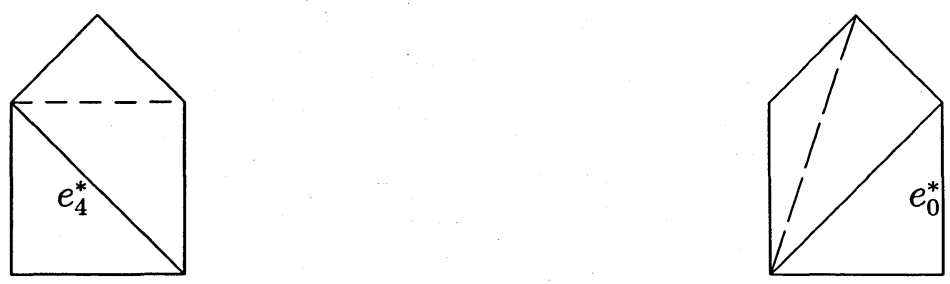

$\Delta\left(G^{2}\right)$

$$
\Delta\left(G^{3}\right)
$$

Figure 6 
We shall require these identities below but omit the proofs (which are standard computations of anharmonic ratios).

Now returning to the previous computation, we have

$$
\begin{aligned}
\beta\left[G_{2}\right] & =-2 \sum_{i=0}^{4} \varphi\left(\lambda_{i}\right) \\
& =-\frac{5 \pi^{2}}{3}-4 \sum_{i=0}^{4} L\left(\lambda_{i}\right),
\end{aligned}
$$

where $L$ denotes Dupont's version [Du] of Rogers' function (so $\varphi \equiv 2 L+\frac{\pi^{2}}{6}$ ). Recall that the functional equation on $L$ is

$$
\begin{aligned}
& L(x)+L(1-x)=-\frac{\pi^{2}}{6} \\
& \sum_{i=0}^{4}(-1)^{i} L\left(\mu_{i}^{-1}\right)=0
\end{aligned}
$$

where $\mu_{i}=\left\{v_{0}: \cdots: \widehat{v_{i}}: \cdots: v_{4}\right\}$.

On the other hand, Lemma 5.2 gives

$$
\begin{aligned}
& \lambda_{i}=1-\mu_{i}^{-1} \text { for } i=0,2,4, \\
& \lambda_{i}=\mu_{i}^{-1} \text { for } i=1,3
\end{aligned}
$$

so we conclude that

$$
\begin{aligned}
\beta\left[G_{2}\right] & =-\frac{5 \pi^{2}}{3}-4\left\{-\frac{\pi^{2}}{2}-\sum_{i=0}^{4}(-1)^{i} L\left(\mu_{i}^{-1}\right)\right\} \\
& =2 \pi-\frac{5 \pi^{2}}{3} \\
& =\frac{\pi^{2}}{3} .
\end{aligned}
$$

Finally,

$$
\begin{aligned}
\int_{\mathcal{M}} \omega \wedge \eta & =\frac{\pi^{2}}{3} \sum_{\left[G_{2}\right] \in\left[W_{2}\right]} \frac{1}{\# A u t\left[G_{2}\right]} \int_{\mathcal{D}\left(G_{2}\right)} \eta \\
& =\frac{\pi^{2}}{3} \int_{\left[W_{2}\right]} \eta,
\end{aligned}
$$

with $\mathcal{D}\left(G_{2}\right)$ given its canonical orientation as in $\S 4$, where the second equality follows from a final application of Theorem $D$. 
This completes the proof in case $F_{g}^{s}$ is different from $F_{1}^{1}, F_{0}^{4}, F_{1}^{2}$. For $F_{1}^{1}$ and $F_{0}^{4}$ (whose moduli spaces are of complex dimension one), we have $W_{2}=\emptyset$, and our argument above shows that $\int_{\mathcal{M}} \omega \wedge \eta=0$ for any compactly supported zeroform $\eta$. For $F_{1}^{2}$, one checks easily (or see [P3, Table 1]) that there is exactly one hyperelliptic fatgraph in codimension 0,1 , or 2 , it occurs in codimension 2 , and it is of type (a) above. The same argument therefore applies, and the proof is complete.

\section{Closing Remarks}

A natural extension of Witten's conjecture in the introduction is that the closure of the cycle $\left[W_{2 i}\right]$ in $\overline{\mathcal{M}}$ actually represents a multiple of the Poincaré dual of $\bar{\kappa}_{i}$, for each $i$. One might also reformulate the problem and consider some other orbifold compactification $\mathcal{M}^{\prime}$ of $\mathcal{M}$ which admits a natural continuous map $\mathcal{M}^{\prime} \rightarrow \overline{\mathcal{M}}$. Letting $\kappa_{i}^{\prime} \in H^{2 i}\left(\mathcal{M}^{\prime}, Q\right)$ denote the corresponding pull-back of $\bar{\kappa}_{i}$ for each $i$, one seeks a compactification $\mathcal{M}^{\prime}$ so that the closure $\left[W_{2 i}^{\prime}\right]$ of $\left[W_{2 i}\right]$ in $\mathcal{M}^{\prime}$ is a cycle representing a multiple of the Poincare dual of $\kappa_{i}^{\prime}$ for each $i$.

In fact, we have studied a particular compactification which arises quite naturally and next briefly survey this work-in-progress to discuss certain conjectures in the current context. We shall limit the discussion here to the case of once-punctured surfaces just for simplicity. In this case, the decorated Teichmüller space $\tilde{\mathcal{T}} \approx \mathcal{T} \times R$ is a product, where $\mathcal{T}$ denotes the Teichmüller space. By definition of lambda lengths and homogeneity of our formulas, the coordinates on $\tilde{\mathcal{T}}$ descend to projective coordinates on $\mathcal{T}$, and, what is more, the "cell-decomposition" of $\tilde{\mathcal{T}}$ descends to a "cell-decomposition" of $\mathcal{T}$.

There is thus an embedding $\mathcal{T} \rightarrow \mathcal{A}$, where $\mathcal{A}$ is the simplicial complex (considered in [Ha]) consisting of all projectively weighted familes of isotopy classes of disjointly embedded essential arcs in the surface with endpoints at the puncture. $\mathcal{A}$ itself is therefore simply the natural simplicial completion of $\mathcal{T} \subset \mathcal{A}$.

The mapping class group acts on $\mathcal{A}$ in the natural way, and we let $\mathcal{M}^{\prime}$ denote the quotient. Simplices in $\mathcal{A}-\mathcal{T}$ have infinite isotropy. As alluded to in the Remark following Theorem D in Section 1 above, the second barycentric 
subdivision of $\mathcal{A}$ descends to a true simplicial complex on $\mathcal{M}^{\prime}$.

Conjecture 6.1. $\mathcal{M}^{\prime}$ is an orbifold.

In the setting of planar surfaces (for which the simplicial completion of $\mathcal{T}$ is not all of $\mathcal{A}$ and we do not here define $\mathcal{M}^{\prime}$ ), we have proved the corresponding conjecture using a rather involved argument.

It is a non-trivial geometric fact that there is actually a natural continuous mapping $\mathcal{M}^{\prime} \rightarrow \overline{\mathcal{M}}$, and we let $\kappa_{i}^{\prime} \in H^{2 i}\left(\mathcal{M}^{\prime}, Q\right)$ denote the pull-back of $\bar{\kappa}_{i}$ for each $i$. Furthermore, let $W_{2 i}^{\prime}$ denote the collection of simplices of codimension $2 i$ in $\mathcal{A}$ defined in analogy to $W_{2 i}$. Each simplex in $W_{2 i}^{\prime}$ admits a canonical orientation just as before, and the associated chain $W_{2 i}^{\prime}$ on $\mathcal{A}$ is again a cycle of non-compact support. This cycle is evidently invariant under the action of the mapping class group, and hence $W_{2 i}^{\prime}$ descends to a rational cycle $\left[W_{2 i}^{\prime}\right]$ of compact support on $\mathcal{M}^{\prime}$.

ConjeCture 6.2. $\left[W_{2 i}^{\prime}\right]$ is a constant multiple of the Poincare dual of $\kappa_{i}^{\prime}$ on $\mathcal{M}^{\prime}$, for each $i \geq 1$.

We mention parenthetically that in the case of the once-punctured torus $F_{1}^{1}$, we have $\mathcal{M}^{\prime}=\overline{\mathcal{M}}$, and the Poincaré dual of the Weil-Petersson two-form is $\pi^{2} / 6$ times the class of the ideal point, where the extra factor of two here (in comparison to Theorem 5.1) comes from the hyperelliptic involution. Thus, both of the conjectures above hold for the surface $F_{1}^{1}$.

This completes the discussion of Theorem 5.1 in the context of our workin-progress on the compactification $\mathcal{M}^{\prime}$ of $\mathcal{M}$, and we turn finally to other closing remarks.

It is appealing to believe that the computational scheme employed here might be a useful tool for deriving analogues of Rogers' function and its AbelSpence functional equation for higher polylogarithms. Of course, a fundamental ingredient for us is an explicit hyperbolic formula for the Weil-Petersson Kähler two-form (namely, the formula in Theorem A, which, in turn, depends on [W1]), and the lack of such a formula for the higher Miller-Morita-Mumford classes could be a basic obstruction to such a dream. 
We finally mention that $[\mathrm{Ko}]$ has given explicit and simple formulas in the simplicial coordinates for Witten's closely related "visible" cohomology classes [Wi] on $\mathcal{M}$, and he has used the integration scheme described herein to explicitly compute their various correlations; one might also use the computational scheme of this paper to find the compact Poincaré duals of these classes.

\section{REFERENCES}

[BE] Bowditch, B. E., and Epstein,D. B. A., Natural Triangulations Associated to a Surface, Topology 27 (1988), 91-117.

[Du] Dupont, J. L., The Dilogarithm as a Characteristic Class for Flat Bundles, Jour. of Pure and Appl. Alg. 44 (1987), 137-164.

[DM] Deligne, P., and Mumford, D., Irreducibility of the Space of Curves of a given Genus, Inst. Hautes Études Sci. Publ. Math. 36 (1979), 75-110.

[Ha] Harer, J. L., Stability of the Homology of the Mapping Class Group, Ann. Math. 121 (1985), 215-249.

[HM] Hain, R. M., and MacPherson, R., Higher Logarithms, Ill, Jour. Math. 34 (1990), 392-475.

[Ko] Kontsevich, M., Intersection Theory on the Moduli Space of Curves and the Matrix Airy Function, Comm. Math. Phys. 147 (1992), 1-23.

[Mi] Miller, E., The Homology of the Mapping Class Group, J. Differetial Geom. 24 (1986), 1-14.

[Mo] Morita, S., Characteristic Classes of Surface Bundles, Invent. Math. 90 (1987), 551-577.

[Mu] Mumford, D., Towards an Enumerative Geometry of the Moduli Space of Curves, Arithmetic and Geometry, Progr. Math. 36 (1983), Birkhäuser, Boston, 271-328.

[P1] Penner, R. C., The Decorated Teichmüller Space of a Punctured Surface, Comm. Math. Phys. 113 (1987), 299-339.

[P2] _ - Perturbative Series and the Moduli Space of Riemann Surfaces, J. Differential Geom. 27 (1988), 35-53.

[P3] _ Weil-Petersson Volumes, J. Differential Geom. 35 (1992), 559-608.

[P4] - The Moduli Space of Punctured Surfaces, in the volume "Mathematical Aspects of String Theory", Advanced Series in Mathematical Physics 1 (1987), World Scientific Publ. Co., Singapore, 313-340, edited by S. T. Yau.

[Wi] Witten, E., Two-dimensional Gravity and Intersection Theory on the Moduli Space, Surveys in Diff. Geom. 1 (1991), 243-310.

[W1] Wolpert, S. A., On the Symplectic Geometry of Deformations of a Hyperbolic Surface, Ann. Math. 117 (1983), 207-234.

[W2] — The Hyperbolic Metric and the Geometry of the Universal Curve, J. Differential Geom. 31 (1990), 417-472.

University of Southern California, Los Angeles, U. S. A.

RECEIVED MAY 6, 1992 